\title{
4 \\ IMPLICACIÓN ESCOLAR DE ESTUDIANTES DE SECUNDARIA: LA INFLUENCIA DE LA RESILIENCIA, EL AUTOCONCEPTO Y EL APOYO SOCIAL PERCIBIDO
}

(SCHOOL ENGAGEMENT IN STUDENTS OF COMPULSORY SECONDARY
EDUCATION: THE INFLUENCE OF RESILIENCE, SELF-CONCEPT AND
PERCEIVED SOCIAL SUPPORT)

Arantzazu Rodríguez-Fernández

Estibaliz Ramos-Díaz

Iker Ros Martínez de Lahidalga

Ana Zuazagoitia Rey-Baltar

Universidad del País Vasco/Euskal Herriko Unibertsitatea (UPV/EHU)

DOI: 10.5944/educXX1.20177

\section{Cómo referenciar este artículo/How to reference this article:}

Rodríguez-Fernández, A.; Ramos-Díaz, E.; Ros, I. y Zuazagoitia, A. (2018). Implicación escolar de estudiantes de secundaria: La influencia de la resiliencia, el autoconcepto y el apoyo social percibido. Educación XX1, 21(1), 87-108, doi: 10.5944/educXX1.20177

Rodríguez-Fernández, A.; Ramos-Díaz, E.; Ros, I. \& Zuazagoitia, A. (2018). Implicación escolar de estudiantes de secundaria: La influencia de la resiliencia, el autoconcepto y el apoyo social percibido. [School engagement in students of compulsory secondary education: The influence of resilience, self-concept and perceived social support]. Educación XX1, 21(1), 87-108, doi: 10.5944/ educXX1.20177

\section{RESUMEN}

El objetivo de este estudio fue doble: en primer lugar, analizar las relaciones entre la implicación escolar y la resiliencia, el autoconcepto y el apoyo social percibido. En segundo lugar, determinar variables predictoras de la implicación escolar. Participaron en el estudio 1250 estudiantes entre 12 y 15 años $(M=13.72, D T=1.09)$, de los cuales 612 eran chicos (49\%) y $638(51 \%)$ chicas, seleccionados aleatoriamente en el País Vasco. Se administraron los siguientes instrumentos de evaluación: Cuestionario de Implicación Escolar (SEM) de Fredericks, Blumenfeld, Friedel, y Paris (2005), Escala de Resiliencia (CDRISC) de Connor y Davidson (2003), Cuestionario de Autoconcepto Dimensional-33 (AUDIM-33) de Fernández-Zabala, Goñi, Rodríguez-Fernández y Goñi (2015), y Cuestionario de Apoyo Familiar y de Amigos (AFA-R) de González 
y Landero (2014). Se hallaron correlaciones significativas entre implicación escolar y resiliencia, autoconcepto y apoyo social. Todas las variables estudiadas fueron predictoras de las tres dimensiones de implicación escolar (conductual, emocional y cognitiva) a excepción del autoconcepto, que se mostró como variable predictora de la implicación conductual y la implicación emocional, pero no de la implicación cognitiva.

\section{PALABRAS CLAVE}

Implicación escolar; resiliencia; autoconcepto; apoyo social percibido; adolescentes.

\section{ABSTRACT}

The aim of this study was twofold: first, to analyze the relationship between school engagement and resilience, self-concept and perceived social support. Second, to determine predictors of school engagement. The sample was comprised of 1,250 students between 12 and 15 years of age $(M=13.72, S D=$ 1.09), of which 612 were boys (49\%) and $638(51 \%)$ girls, randomly selected in the Basque Country. The following assessment instruments were administered: School Engagement Measurement (SEM) of Fredericks, Blumenfeld, Friedel and Paris (2005); Resilience Scale (CD-RISC) of Connor and Davidson (2003); Dimensional Self-Concept Questionnaire-33 (AUDIM-33) of Fernández-Zabala, Goñi, Rodríguez-Fernández and Goñi (2015); and Social Support from Family and Friends Questionnaire (AFA-R) of González and Landero (2014). Significant correlations between school engagement and resilience, self-concept and social support were found. All the variables studied were predictive of the three dimensions of school engagement (behavioral, emotional and cognitive) with the exception of self-concept, which was found to be a predictor of behavioral engagement and emotional engagement, but not of cognitive engagement.

\section{KEY WORDS}

School responsability; resilience; self-concept; perceived social support; adolescents.

\section{INTRODUCCIÓN}

En los últimos años, la preferencia por el estudio de las fortalezas y las cualidades positivas del alumnado frente al enfoque del déficit ha experimentado un gran auge dentro de la psicología de la educación (Kristjáns- 
son, 2012). Entre dichas cualidades sobresale la implicación escolar (school engagement), variable que es identificada como un elemento crucial para el desarrollo psicosocial y el éxito académico (Motti-Stefadini y Masten, 2013; Ramos-Díaz, Rodríguez-Fernández, Fernández-Zabala, Revuelta, y Zuazagoitia, 2016). Concretamente, en el periodo de la adolescencia la implicación del estudiante con la escuela adopta un significado fundamental en la comprensión del comportamiento saludable (Li y Lerner, 2011). El constructo de implicación escolar no cuenta con una acotación conceptual unívoca y es debatido por los investigadores (Appleton, Christenson, y Furlong, 2008; Lawson y Lawson, 2013), si bien puede definirse como el grado en que el alumnado se compromete con la escuela y está motivado para aprender (Simons-Morton y Chen, 2009), es decir, es un meta-constructo que incluye tres dimensiones: cognitiva, emocional y conductual (Appleton, 2012; Fredricks, Blumenfeld, y Paris, 2004; Glanville y Wildhagen, 2007; González y Verónica-Paoloni, 2014; Veiga, Burden, Appleton, Céu, y Galvao, 2014). Por tanto, en el presente trabajo se conceptualiza la implicación escolar como una variable multidimensional que refleja cada uno de los subtipos propuestos por Fredricks et al. (2004): participación en la escuela (implicación conductual), identificación con la escuela (implicación emocional) e inversión en la tarea de aprendizaje (implicación cognitiva). La investigación reciente ha ido proporcionando progresivamente evidencia empírica sobre la existencia de condiciones psicológicas y ambientales que influyen sobre la implicación con el centro educativo (Christenson, Reschly, y Wylie, 2012).

Precisamente, entre los factores psicológicos que afectan a la implicación escolar se encuentra la resiliencia, concepto científico que ha ido ganando un considerable protagonismo en el ámbito de la psicología de la educación debido al importante papel de la escuela como promotora de bienestar (Toland y Carrigan, 2011). Son numerosas las interpretaciones que en los últimos tiempos se vienen haciendo sobre este constructo y, aunque existe una falta de consenso en su definición (Fletcher y Sarkar, 2013), hay un creciente acuerdo en definir la resiliencia como el logro de una adaptación positiva. Dicho con otras palabras, la resiliencia sería el afrontamiento adecuado que hace una persona durante su desarrollo vital ante la presencia de adversidades o de una exposición importante a algún tipo de riesgo que podría conducir a la persona con mucha probabilidad hacia el desajuste psicosocial (Luthar, Cicchetti, y Becker, 2000; Masten, 2014; Rutter, 2007; Ungar, 2011). En la etapa de la adolescencia, la resiliencia implica superar los potenciales efectos negativos producidos por la exposición al riesgo mediante estrategias positivas de fortalecimiento y afrontamiento (Fergus y Zimmerman, 2005). Los antecedentes de investigación que se aproximan al análisis de la relación entre la resiliencia y la implicación escolar son escasos (Jones y Lafreniere, 2014; Rodríguez-Fernández, Ramos-Díaz, Ros, Fernández-Zabala, y Revuelta, 2015; Ungar y Liebenberg, 2013), aunque 
al amparo de la psicología positiva han surgido estudios que vinculan a la resiliencia con otros factores y variables educativas tales como el clima motivacional del aula (Alonso-Tapia, Nieto, y Ruiz, 2013), el uso de diversas estrategias motivacionales y de regulación emocional (Fried y Chapman, 2012) o el rendimiento en estudiantes adolescentes (Gaixola, Lugo, Guedea, y Villa, 2013; Gaixola, Lugo, y Villa, 2013; Villalta, 2010). Por lo tanto, y aunque se desconoce en buena medida por el momento si la resiliencia es un predictor estable de un mayor nivel de implicación escolar, cabe figurarse una correlación positiva entre ambas variables, de manera que los/las estudiantes que responden de manera resiliente ante los estresores del contexto escolar, podrían desplegar reacciones adaptativas hacia el centro educativo y lo académico, participar en la escuela y dedicar más tiempo a las tareas de aprendizaje que los/las estudiantes no resilientes.

Otro factor intrapersonal que influye en la adaptación escolar de los adolescentes es el autoconcepto, definido como el conjunto de percepciones que una persona mantiene sobre sí misma a partir de la valoración personal y de la evaluación de los otros significativos (Shalvenson, Hubner, y Stanton, 1976). Debido a su gran centralidad psicológica, el autoconcepto ha sido identificado como un constructo teórico estrechamente relacionado con el ajuste escolar (Rodríguez-Fernández, Droguett, y Revuelta, 2012). La evidencia empírica predominante muestra que el autoconcepto guarda una relación directa con diversas variables educativas, tales como el logro académico (Huang, 2011; Preckel, Niepel, Schneider, y Brunner, 2013) o la implicación del alumnado en el proceso de aprendizaje (Inglés, MartínezMonteagudo, García-Fernández, Valle, y Castejón, 2014), y que dicha relación parece estar mediada por la resiliencia (Rodríguez-Fernández et al., 2015). Pero no se ha analizado aún la relación del autoconcepto con cada una de las dimensiones de la implicación escolar.

Un factor de carácter contextual que también está asociado a la implicación escolar es el apoyo social percibido (Lam, Wong, Yang, y Liu, 2012), entendiendo por tal la percepción subjetiva respecto a la adecuación del soporte proporcionado por la red social. Se trata de un fenómeno complejo y dinámico que comprende un conjunto de elementos en interacción y que evoluciona en gran medida a lo largo de la adolescencia (Cohen, 2004). Por un lado, la familia es uno de los sistemas más inmediatos del desarrollo humano (Brofenbrenner, 1986), por lo que es esperable que el apoyo familiar juegue un rol muy importante en la implicación escolar (Veiga et al., 2012). Por otro lado, existen estudios que confirman la relación positiva entre el apoyo de los iguales y la implicación escolar general (Perdue, Manzeske, y Estell, 2009), de manera que cuando los estudiantes tienen amistades y se sienten socialmente conectados, es esperable que estén predispuestos a experimentar sentimientos positivos hacia lo académico y las actividades 
escolares (Juvonen, Espinoza, y Knifsend, 2012). Pero al tomarse en consideración la interacción del apoyo familiar y del grupo de iguales sobre el ajuste escolar, se aporta información sobre la incidencia diferenciada que tienen ambos tipos de apoyo, de manera que el apoyo de los amigos se ve notablemente reducido en comparación con la influencia de la familia (Rodríguez-Fernández et al., 2012; Rodríguez-Fernández, Ramos-Díaz, Madariaga, Arrivillaga, y Galende, 2016).

Por último, apenas se cuenta con trabajos que aborden las diferencias de sexo en implicación escolar (Veiga et al., 2014), habiéndose sugerido diferencias a favor de las chicas adolescentes en comparación con los adolescentes de sexo masculino (Lam et al., 2012). Por lo tanto, resulta interesante analizar empíricamente las diferencias de sexo en la predicción de la implicación escolar y la relación existente entre dichas variables.

Este estudio plantea como objetivo el análisis de la relación que mantienen determinados recursos personales y contextuales con la implicación escolar de los adolescentes (diferenciando ambos sexos), entendiendo la implicación escolar como la medida diferenciada de la implicación conductual, implicación emocional e implicación cognitiva. Se proponen 3 hipótesis:

1. existen correlaciones directas y significativas de la implicación escolar (conductual, emocional y cognitiva) para con la resiliencia, el autoconcepto, y el apoyo social (de la familia y de los amigos) tanto en los adolescentes varones como en las mujeres adolescentes;

2. la correlación entre dichas variables estará mediada por el efecto del sexo;

3. las variables independientes resiliencia, autoconcepto, apoyo familiar y apoyo de amigos, predicen la variable dependiente implicación escolar (conductual, emocional y cognitiva), para ambos sexos.

\section{MÉTODO}

\section{Diseño de investigación}

Dada la imposibilidad de manipular la variable independiente para el estudio aquí presentado, se opta por un diseño correlacional transversal que analice las relaciones entre la variable dependiente (implicación escolar) y las independientes (sexo, autoconcepto, resiliencia y apoyo social de la familia y de los amigos) y dentro de los estudios correlacionales, se opta por el estudio ex post facto retrospectivo de grupo único. Ahora bien, dado que una de las hipótesis a someter a prueba presupone que la implicación 
escolar estará predicha por las variables independientes, también se realiza un diseño transversal causal no experimental. No obstante, cabe aclarar que a pesar de que en esta investigación se realizan análisis estadísticos destinados a conocer posibles variables predictoras de la variable dependiente implicación escolar, no se puede hablar de causalidad pura, pues para ello se debería realizar un estudio longitudinal experimental y no un diseño transversal causal no experimental. Es decir, cuando en este trabajo se habla de predicción y causalidad, se hace referencia a una predicción y causalidad a niveles puramente estadísticos.

\section{Variables e instrumentos}

Las variables de este estudio fueron un total de 5: la implicación escolar, la resiliencia, el autoconcepto general, el apoyo social de los padres y el apoyo social de los amigos. Para evaluar la implicación escolar se utilizó el Cuestionario de Implicación Escolar (SEM) de Fredericks, Blumenfeld, Friedel y Paris (2005), que está compuesto por 19 ítems (ejemplos: Me divierto estando en clase; Presto atención en clase), con un formato de respuesta Likert de 5 grados (donde $1=$ nunca y $5=$ siempre). El análisis factorial de los elementos en la versión original da lugar a tres escalas que evalúan la implicación conductual (5 ítems), la implicación emocional (6 ítems) y la implicación cognitiva (8 ítems). Los autores informan de múltiples análisis que confirman la validez de la versión inglesa del cuestionario (Blumenfeld et al., 2005; Fredricks et al., 2005; Fredricks y McColskey, 2012). En la muestra de esta investigación se obtuvieron índices de consistencia interna de $\alpha=.81$ para la escala de implicación emocional, de $\alpha=.74$ para la implicación conductual y de $\alpha=.77$ para la implicación cognitiva.

La resiliencia fue medida mediante la Escala de Resiliencia ConnorDavidson (CD-RISC), de Connor y Davidson (2003), compuesta por un total de 25 ítems (ejemplos: Soy capaz de adaptarme a los cambios; Puedo alcanzar mis metas) y se responde en una escala Likert que oscila desde $0=n a d a$ de acuerdo a 4=totalmente de acuerdo. El análisis factorial original sugiere una estructura de cinco factores (competencia personal, confianza en la intuición y tolerancia a la adversidad, aceptación positiva del cambio, control y espiritualidad), aunque siguiendo las recomendaciones de otros autores (Campbell-Sills, Cohan, y Stein, 2006) se tomó la puntuación total de los ítems. Las características psicométricas de CD-RISC, en un estudio preliminar en población general y muestra clínica, indicaron una adecuada consistencia interna, fiabilidad test-retest, validez convergente y divergente (Connor y Davidson, 2003), y buena validez de constructo en población adolescente (Jorgensen y Seedat, 2008; Yu, Lau, Mak, Zhang, y Lui, 2011). Para 
precisar la versión final de la escala se tuvo en cuenta la versión traducida al castellano proporcionada por los autores originales (Bobes, Bascaran, García-Portilla, Bousoño, Sáiz, y Wallance, 2001). La consistencia interna alcanzada en este estudio fue elevada $(\alpha=.86)$.

El Cuestionario de Autoconcepto Multidimensional-33 (AUDIM-33) de Fernández-Zabala, Goñi, Rodríguez-Fernández y Goñi (2015) se empleó para obtener una medida del autoconcepto. Posee un formato de respuesta para sus 33 ítems en escala Likert de 5 grados que oscila desde 1=falso a $5=$ verdadero. Consta de doce escalas que evalúan las dimensiones del autoconcepto, además de una escala general para la medida del autoconcepto general. Cada una de las escalas de señalan a continuación: autoconcepto general, autoconcepto académico verbal, autoconcepto académico matemático, habilidad física y condición física, atractivo físico, fuerza física, honradez, ajuste emocional, autonomía, autorrealización, responsabilidad social y competencia social. En los análisis se utilizó únicamente la dimensión general del autoconcepto, compuesta por 5 ítems (ejemplos: Me siento a disgusto conmigo mismo/a; Me siento una persona afortunada). Respecto a las propiedades psicométricas, la consistencia alfa del cuestionario con la muestra del presente estudio fue $\alpha=.82$.

El apoyo social se evaluó a través del Cuestionario de Apoyo Social Familiar y de Amigos (AFA-R) de González y Landero (2014), compuesto por 14 ítems (ejemplos: Cuentas con alguien de tu familia que te ayude a resolver algún problema; Confías en algún amigo/a para hablar de las cosas que te preocupan) destinados a medir dos dimensiones: el apoyo familiar ( 7 ítems) y el apoyo de los amigos ( 7 ítems). El formato de respuesta corresponde a una escala tipo Likert de 5 alternativas de respuesta $(1=$ nunca y $5=$ siempre $)$. El índice de consistencia interna obtenido con la muestra del presente estudio fue $\alpha=.84$ para el apoyo familiar y $\alpha=.82$ para apoyo de amigos.

\section{Participantes}

Participan en esta investigación un total de 1250 estudiantes de 12 a 15 años $(M=13.72 ; D T=1.09)$, de los cuales $612(49 \%)$ eran chicos y $638(51 \%)$ chicas. La selección de los participantes se llevó a cabo mediante muestreo aleatorio de estudiantes de secundaria escolarizados en centros educativos de la Comunidad Autónoma del País Vasco (CAPV). La distribución de la muestra en los distintos niveles educativos fue estadísticamente proporcionada (ver tabla 1) ya que la ji-cuadrado de Pearson indicó que no había diferencias en la distribución del número de participantes de cada sexo en los diferentes niveles educativos $\left(\chi_{(1)}^{2}=4.66, p>.05\right)$. 
ARANTZAZU RODRÍGUEZ-FERNÁNDEZ, ESTIBALIZ RAMOS-DÍAZ, IKER ROS MARTÍNEZ DE LAHIDALGA Y ANA ZUAZAGOITIA REY-BALTAR

Tabla 1

Distribución de la Muestra por Sexo y Edad

\begin{tabular}{lccccc}
\hline \multicolumn{1}{c}{ Sexo } & \multicolumn{2}{c}{ Edad } & Total \\
\hline & $\mathbf{1 .}^{\mathbf{0}}$ ESO & $\mathbf{2 .}^{\mathbf{0}}$ ESO & $\mathbf{3 .}^{\mathbf{0}}$ ESO & $\mathbf{4 .}^{\mathbf{0}}$ ESO & \\
\hline Hombre & $114(9.1 \%)$ & $118(9.4 \%)$ & $182(14.6 \%)$ & $198(15.8 \%)$ & $612(49.0 \%)$ \\
Mujer & $118(9.4 \%)$ & $154(12.3 \%)$ & $171(13.7 \%)$ & $195(15.6 \%)$ & $638(51.0 \%)$ \\
Total & $232(18.6 \%)$ & $272(21.8 \%)$ & $353(28.2 \%)$ & $393(31.4 \%)$ & $1250(100 \%)$ \\
\hline
\end{tabular}

Nota. $\chi^{2}=4.66, p<.05$

\section{PROCEDIMIENTO}

De un total de 350 centros de educación secundaria, 9 instituciones escolares ( 4 de titularidad concertada y 5 de titularidad pública) fueron seleccionadas aleatoriamente del listado de centros educativos de la CAPV. Con el propósito de presentar la investigación y solicitar la participación voluntaria en la misma se contactó con los centros seleccionados. Una vez confirmada su participación, se entregaron los consentimientos informados para los tutores. Se administraron los instrumentos de evaluación a los estudiantes dentro del horario de clase y de manera simultánea a todo el alumnado integrante de un mismo aula, siendo el periodo de respuesta medio de treinta minutos aproximadamente. A fin de mitigar respuestas en dirección de las hipótesis de la investigación, se empleó el criterio de ciego único, evitando que los estudiantes conocieran la finalidad del estudio. La confidencialidad y voluntariedad fueron asimismo garantizadas para reducir los efectos de la deseabilidad social.

\section{Análisis de datos}

Con la finalidad de analizar las relaciones que existen entre el apoyo social de la familia y los amigos, la resiliencia, el autoconcepto y la implicación escolar, se calcularon los coeficientes de correlación de Pearson de orden cero con las puntuaciones obtenidas en los cuestionarios AFA-R, CD-RISC, AUDIM-33 y SEM. Ahora bien, para conocer el efecto mediador de la variable sexo sobre la relación anterior, se procede a calcular los coeficientes de correlación parcial que permitirán su comparación con los valores de las correlaciones de orden cero. En tercer lugar, con el objetivo de identificar variables predictoras de la implicación escolar se realizó un análisis de regresión lineal múltiple, método de pasos sucesivos, de forma 
que cuando alguna variable resulta no predictora es sacada e introducida con el resto sucesivamente en el modelo de regresión hasta obtener el mejor ajuste de predicción posible. Los análisis estadísticos señalados fueron realizados con el paquete estadístico SPSS 22.0 para Windows. Para el tratamiento de los valores perdidos se extrajo, a partir del algoritmo de maximización de expectativa y de la Cadena Markov Monte Carlo (MCMC), una puntuación aproximada al ítem perdido basada en el patrón de respuestas.

\section{RESULTADOS}

\section{Relaciones de la implicación escolar con resiliencia, autoconcepto y apoyo social}

Se calcularon, de un lado, los coeficientes de correlación de Pearson entre las dimensiones de la implicación escolar (implicación conductual, implicación emocional e implicación cognitiva) y las variables resiliencia, autoconcepto general y apoyo social percibido tanto para la muestra total como para ambos sexos de manera diferenciada, y, de otro lado, las correlaciones parciales controlando la variable sexo. Los resultados se exponen en la Tabla 2.

Tabla 2

Correlaciones de Pearson entre las Dimensiones de la Implicación Escolar, la Resiliencia, el Autoconcepto y el Apoyo Social, y Correlaciones Parciales controlando el Efecto del Sexo

\begin{tabular}{|c|c|c|c|c|}
\hline & $\begin{array}{l}\text { Muestra total } \\
\quad(\mathbf{N}=1250)\end{array}$ & $\begin{array}{l}\text { Muestra total con- } \\
\text { trolando el efecto } \\
\text { del sexo }(N=1250)\end{array}$ & $\begin{array}{l}\text { Hombre } \\
(N=612)\end{array}$ & $\begin{array}{c}\text { Mujer } \\
(\mathrm{N}=638)\end{array}$ \\
\hline & \multicolumn{4}{|c|}{ Implicación conductual } \\
\hline Resiliencia & $.232 * *$ & $.273 * *$ & $.225 * *$ & $.321 \%$ \\
\hline Autoconcepto & $.249 * *$ & $.276 * *$ & $.232 * *$ & $.318^{* *}$ \\
\hline Apoyo familiar & $.298 * *$ & $.295 * *$ & $.285 * *$ & $.306 * *$ \\
\hline \multirow[t]{2}{*}{ Apoyo amigos } & $.065^{*}$ & .017 & .065 & -.035 \\
\hline & \multicolumn{4}{|c|}{ Implicación emocional } \\
\hline Resiliencia & $.329 * *$ & $.368 * *$ & $.309 * *$ & $.426 \%$ \\
\hline Autoconcepto & $.345 * *$ & $.371 \% *$ & $.295 * *$ & $.440 \% *$ \\
\hline Apoyo familiar & $.328 * *$ & $.326^{* * *}$ & $.285 * *$ & $.366^{* *}$ \\
\hline Apoyo amigos & $.224 *$ & $.191 \% *$ & $.166 * *$ & $.219 * *$ \\
\hline
\end{tabular}




\begin{tabular}{lcccc}
\hline & $\begin{array}{c}\text { Muestra total } \\
(\mathbf{N}=\mathbf{1 2 5 0})\end{array}$ & $\begin{array}{c}\text { Muestra total con- } \\
\text { trolando el efecto } \\
\text { del sexo }(\mathbf{N}=\mathbf{1 2 5 0})\end{array}$ & $\begin{array}{c}\text { Hombre } \\
(\mathbf{N}=\mathbf{6 1 2})\end{array}$ & $\begin{array}{c}\text { Mujer } \\
\mathbf{( N = 6 3 8 )}\end{array}$ \\
\hline Resiliencia & $.309^{* *}$ & \multicolumn{2}{c}{ Implicación cognitiva } \\
Autoconcepto & $.160^{* *}$ & $.316^{* *}$ & $.301 * *$ & $.330^{* *}$ \\
Apoyo familiar & $.260^{* *}$ & $.161^{* *}$ & $.167^{* *}$ & $.159 * *$ \\
Apoyo amigos & .035 & $.260^{* *}$ & $.235^{* *}$ & $.284^{* *}$ \\
\hline
\end{tabular}

$* * p<.001, * p<.05$

En primer lugar, los coeficientes de Pearson reflejan, tanto para la muestra total como para las muestras en función del sexo, interrelaciones significativas entre las variables psicológicas (resiliencia y autoconcepto) y las tres dimensiones de la implicación escolar al nivel de $p<.001$ o $p<.05$, si bien son algo más altas en la muestra de chicas que en la de chicos. Es decir, los estudiantes resilientes y con autoconcepto positivo estarán más implicados escolarmente, y al revés, y especialmente en el caso de las chicas. Respecto al apoyo social, los resultados también evidencian tanto en la muestra general como diferenciada por sexos, correlaciones positivas del apoyo familiar con implicación conductual, implicación emocional e implicación cognitiva, mientras que el apoyo de amigos solo se encuentra conectado significativamente con la dimensión emocional de la implicación. En segundo lugar, las correlaciones parciales controlando el efecto del sexo muestran resultados similares y con muy poca variación con respecto a las de orden cero, lo que significa que el sexo, aunque posee un efecto mediador estadístico en su relación con las variables objeto de estudio, es un efecto leve.

Se confirma, por tanto, la hipótesis de que la implicación escolar guarda una relación directa con la resiliencia, el autoconcepto y el apoyo social, considerando la excepción del apoyo de amigos, que solo mantiene correspondencia con la dimensión emocional de la implicación escolar. También se ve confirmada la hipótesis que defiende que el sexo posee un efecto estadístico mediador en la relación entre la implicación y las otras cuatro variables estudiadas en esta investigación.

\section{Variables predictoras de la implicación escolar}

Con el objetivo de identificar las variables que predicen una alta puntuación en implicación escolar, se realizó un análisis de regresión múltiple, método de pasos sucesivos (ver Tabla 3). En este análisis se introdujeron 
ARANTZAZU RODRÍGUEZ-FERNÁNDEZ, ESTIBALIZ RAMOS-DÍAZ, IKER ROS MARTÍNEZ DE LAHIDALGA

como variables dependientes las 3 dimensiones de la implicación escolar (conductual, emocional y cognitiva), y como variables predictoras la resiliencia, el autoconcepto, el apoyo familiar y el apoyo de los amigos.

Tabla 3

Variables Predictoras de Implicación Conductual, Implicación Emocional e Implicación Cognitiva

\begin{tabular}{|c|c|c|c|c|c|c|c|}
\hline & $\begin{array}{c}R^{2} \\
\text { acumu- } \\
\text { lado }\end{array}$ & $\begin{array}{c}\Delta R^{2} \\
\text { acumu- } \\
\text { lado }\end{array}$ & $\boldsymbol{B}$ & Constante & $\beta$ & $t$ & $p$ \\
\hline \multicolumn{8}{|c|}{ Implicación conductual } \\
\hline \multicolumn{8}{|l|}{ Muestra total } \\
\hline Apoyo familiar & .298 & .089 & .142 & 13.61 & .245 & 8.23 & $.000 * * *$ \\
\hline Resiliencia & .336 & .113 & .040 & 10.61 & .124 & 4.13 & $.000 * * *$ \\
\hline Autoconcepto & .350 & .123 & .106 & 10.18 & .122 & 3.94 & $.000 * * *$ \\
\hline Apoyo amigos & .357 & .127 & -.048 & 10.87 & -.074 & -2.57 & $.010 * * *$ \\
\hline \multicolumn{8}{|l|}{ Hombres } \\
\hline Apoyo familiar & .285 & .081 & .152 & 13.08 & .250 & 5.80 & $.000 * * *$ \\
\hline Resiliencia & .325 & .105 & .047 & 9.79 & .139 & 3.24 & $.001 * * *$ \\
\hline Autoconcepto & .339 & .115 & .113 & 9.08 & .116 & 2.68 & $.008 * * *$ \\
\hline Apoyo amigos & .351 & .123 & -.067 & 9.69 & -.100 & -2.36 & $.018 * * *$ \\
\hline \multicolumn{8}{|l|}{ Mujeres } \\
\hline Apoyo familiar & .387 & .147 & .122 & 10.24 & .228 & 5.75 & $.000 * * *$ \\
\hline Resiliencia & .321 & .101 & .065 & 12.74 & .216 & 5.32 & $.000 * * *$ \\
\hline Apoyo amigos & .427 & .179 & -.152 & 12.45 & -.226 & -5.90 & $.000 * * *$ \\
\hline Autoconcepto & .459 & .206 & .156 & 12.39 & .201 & 4.75 & $.000 * * *$ \\
\hline \multicolumn{8}{|c|}{ Implicación emocional } \\
\hline \multicolumn{8}{|l|}{ Muestra total } \\
\hline Autoconcepto & .345 & .119 & .212 & 13.48 & .177 & 5.97 & $.000 * * *$ \\
\hline Apoyo familiar & .409 & .167 & .149 & 9.33 & .188 & 6.58 & $.000 * * *$ \\
\hline Resiliencia & .441 & .195 & .079 & 5.70 & .180 & 6.26 & $.000 * * *$ \\
\hline Apoyo amigos & .447 & .200 & .073 & 4.66 & .081 & 2.95 & $.003 * * *$ \\
\hline \multicolumn{8}{|l|}{ Hombres } \\
\hline Resiliencia & .309 & .094 & .091 & 10.25 & .197 & 4.76 & $.000 * * *$ \\
\hline Apoyo familiar & .374 & .137 & .153 & 6.29 & .183 & 4.59 & $.000 * * *$ \\
\hline Autoconcepto & .398 & .154 & .206 & 4.94 & .153 & 3.61 & $.000 * * *$ \\
\hline
\end{tabular}




\begin{tabular}{|c|c|c|c|c|c|c|c|}
\hline & $\begin{array}{c}R^{2} \\
\text { acumu- } \\
\text { lado }\end{array}$ & $\begin{array}{c}\Delta R^{2} \\
\text { acumu- } \\
\text { lado }\end{array}$ & $\boldsymbol{B}$ & Constante & $\beta$ & $t$ & $p$ \\
\hline \multicolumn{8}{|l|}{ Mujeres } \\
\hline Autoconcepto & .440 & .194 & .266 & 13.09 & .249 & 6.28 & $.000 * * *$ \\
\hline Resiliencia & .506 & .256 & .104 & 7.27 & .251 & 6.53 & $.000 * * *$ \\
\hline Apoyo familiar & .535 & .286 & .141 & 4.82 & .190 & 5.15 & $.000 * * *$ \\
\hline \multicolumn{8}{|c|}{ Implicación cognitiva } \\
\hline \multicolumn{8}{|l|}{ Muestra total } \\
\hline Resiliencia & .309 & .095 & .166 & 8.53 & .269 & 9.72 & $.000 * * *$ \\
\hline Apoyo familiar & .358 & .127 & .245 & 3.94 & .218 & 7.58 & $.000 * * *$ \\
\hline Apoyo amigos & .369 & .134 & -.122 & 5.76 & -.096 & -3.38 & $.001 \% * *$ \\
\hline \multicolumn{8}{|l|}{ Hombres } \\
\hline Resiliencia & .301 & .091 & .180 & 7.61 & .273 & 6.80 & $.000 * * *$ \\
\hline Apoyo familiar & .341 & .117 & .236 & 3.29 & .198 & 4.71 & $.000 * * *$ \\
\hline Apoyo amigos & .351 & .123 & -.116 & 4.41 & -.088 & -2.09 & $.036 * * *$ \\
\hline \multicolumn{8}{|l|}{ Mujeres } \\
\hline Resiliencia & .330 & .108 & .178 & 8.38 & .296 & 7.61 & $.000 * * *$ \\
\hline Apoyo familiar & .381 & .143 & .252 & 4.02 & .236 & 6.01 & $.000 * * *$ \\
\hline Apoyo amigos & .406 & .161 & -.199 & 7.38 & -.149 & -3.83 & $.000 * * *$ \\
\hline
\end{tabular}

$* p<.05, * * p<.15 * * *, p<.001$

Las cuatro variables predictoras de implicación conductual propuestas resultaron significativas: apoyo familiar $\left(t=8.23^{*}, p<.05\right)$, resiliencia $\left(t=4.13^{*}, p<.05\right)$, autoconcepto $(t=3.94 *, p<.05)$ y apoyo de amigos $\left(t=-2.57^{*}, p<.05\right)$, llegando a explicar entre todas ellas el $12.7 \%$ de la varianza de la implicación conductual. Los coeficientes Beta estandarizados indican que el apoyo familiar es la variable con mayor peso sobre la variable criterio $(\beta=.245)$. También en función del sexo las cuatro variables resultaron significativamente predictoras para la implicación conductual, explicando el $12.3 \%$ de la varianza en el grupo de sexo masculino y el $20.6 \%$ en el caso del sexo femenino. Los coeficientes de regresión estandarizados Beta señalan que, de ellas, el apoyo de la familia es la variable con mayor capacidad predictora tanto en la muestra de chicos $(\beta=.250)$, como en la de chicas $(\beta=.228)$.

Con respecto a la implicación emocional en la muestra total, de nuevo las cuatro variables propuestas como predictoras presentan capaci- 
dad predictiva a nivel significativo (autoconcepto: $t=5.97^{*}, p<.05$; apoyo familiar: $t=6.58^{*}, p<.05$; resiliencia: $t=6.26^{*}, p<.05$; y apoyo de amigos: $t=2.95^{*}, p<.05$ ), si bien los coeficientes de regresión estandarizados indican que apoyo familiar $(\beta=.188)$ es la más predictora seguida por autoconcepto $(\beta=.177)$ y resiliencia $(\beta=.180)$ con niveles muy similares de peso sobre la implicación emocional. Ahora bien, si este análisis se repite de forma diferencial para el sexo, se encuentra, de un lado, que el apoyo de los amigos pierde su peso en la implicación emocional tanto en las chicas como en los chicos y, de otro lado, que según los coeficientes de regresión, la resiliencia pasa a ser la variable más importante en la predicción de implicación en la muestra masculina $(\beta=.197)$ y el autoconcepto en la muestra femenina $(\beta=.249)$. Estas tres variables (apoyo familiar, resiliencia y autoconcepto) explican el $15.4 \%$ de la varianza para los chicos y el $28.6 \%$ para las chicas.

Finalmente, en la implicación cognitiva de las cuatro variables planteadas como sus posibles predictoras, tanto en la muestra total como diferenciada por sexos queda eliminado el autoconcepto, resultando predictores significativos la resiliencia $\left(t=9.72^{*}, p<.05\right)$, el apoyo familiar $\left(t=7.58^{*}, p<.05\right)$ y el apoyo de los amigos $\left(t=-3.38^{*}, p<.05\right)$, con una capacidad explicativa del $13.4 \%$ de la varianza. Los coeficientes Beta revelan que la resiliencia es la variable de mayor peso sobre la implicación cognitiva tanto en la muestra total $(\beta=.269)$, como en la de chicos $(\beta=-.273)$ y en la de chicas $(\beta=.296)$.

Tras estos análisis puede concluirse que, tal y como defiende la tercera hipótesis planteada en el presente estudio, tanto las variables psicológicas (resiliencia y autoconcepto) como las variables de apoyo social (apoyo familiar y apoyo de amigos) poseen capacidad predictiva estadística sobre las dimensiones de la implicación escolar, a excepción del autoconcepto, que no parece tener incidencia significativa sobre la implicación cognitiva si se considera en interacción con la resiliencia y el apoyo social, y el apoyo de los amigos sobre la implicación emocional en la muestra de chicos y de chicas.

\section{DISCUSIÓN}

Considerando desde una perspectiva ecológica la relevancia de los atributos personales y los factores contextuales para lograr un adecuado ajuste en la escuela durante la etapa de Educación Secundaria Obligatoria (Rodríguez-Fernández, et al., 2012), el presente trabajo analiza las relaciones que mantienen determinados aspectos psicológicos (resiliencia y autoconcepto) y ambientales (apoyo social percibido familiar y de amigos) con los com- 
ponentes conductual, emocional y cognitivo de la implicación escolar. La opción de considerar simultáneamente distintos elementos se ajusta adecuadamente a las complejas interacciones persona-contexto que facilitan el bienestar del alumnado, siendo este el camino por el que se precisa avanzar para obtener una explicación cabal de la conducta adolescente (Casas, 2011; Rodríguez-Fernández, et al., 2012).

Con base en los resultados de investigaciones previas que exploran la naturaleza multicausal de la conducta adaptada de escolares adolescentes (Gutiérrez y Romero, 2014; Moreno y Vera, 2011; Rodríguez-Fernández et al., 2012; Rodríguez-Fernández, Ramos-Díaz, Madariaga, et al., 2016), la principal hipótesis de este estudio planteaba que los estudiantes resilientes que manifiestan un buen concepto de sí mismos y perciben un mayor apoyo social de su familia y círculo de amistades, obtendrían mejores puntuaciones en las diferentes dimensiones de la implicación escolar evaluadas. Los resultados apuntan, en la línea de investigaciones previas (Veiga, et al., 2012), a la conexión entre la calidad de las relaciones familiares y la implicación escolar en sus dimensiones conductual, emocional y cognitiva. Ahora bien, no se puede afirmar lo mismo de la relación entre el apoyo de los iguales y la implicación escolar, pues en algunos casos únicamente se encuentran relaciones positivas para con la implicación escolar afectiva (García-Reid, 2007) y la implicación general (Perdue, et al., 2009) y en otros ni siquiera parece existir tal relación (Rodríguez-Fernández, et al. 2012). Los resultados de este trabajo sí encuentran relación entre el apoyo de los pares y la implicación escolar, pero únicamente en su vertiente emocional, tanto en la muestra de los chicos como en la de las chicas adolescentes. Y dichas relaciones se mantienen constantes tanto en la muestra de chicos como en la de chicas a pesar del leve efecto mediador de la variable sexo en la relación entre las dimensiones de la implicación escolar para con el apoyo social.

Ahora bien, no se encuentran muchos estudios que especifiquen el papel que ejercen de manera conjunta el apoyo familiar y el apoyo de amigos sobre las tres dimensiones de la implicación escolar. El estudio de Rodríguez-Fernández, et al. (2012) encontró que el apoyo de la familia presenta un efecto directo sobre el ajuste escolar, mientras que no sucede lo mismo con el apoyo de las amistades. Los resultados del presente estudio, de un lado, ponen de manifiesto la necesidad de tomar en cuenta al mismo tiempo distintas variables contextuales, pues ponen de relieve cambios de relaciones que los análisis bivariados suelen mantener ocultos. Así, en este estudio se comprueba que el examen conjunto de los dos tipos de apoyo (familiar y amigos) reduce la relación bivariada entre el apoyo de los amigos y la implicación afectiva hasta hacerla desaparecer, y hace emerger la importancia de este apoyo sobre la implicación cognitiva y conductual. Se observa que el 
apoyo familiar resulta más relevante en la predicción de la implicación escolar en todas sus facetas, mientras que el apoyo de las amistades se presenta como predictor inverso y mucho más débil de la implicación conductual e implicación cognitiva. Dicho de otro modo, parece que el apoyo del círculo de amistades predice buenos resultados en la identificación del alumnado con el centro educativo, pero incide de manera inversa sobre la participación y la implicación en la tarea de aprendizaje.

El autoconcepto es un constructo relevante en las ciencias sociales y la evidencia empírica predominante muestra que guarda una relación directa con diversas variables educativas, tales como el logro académico (Huang, 2011; Preckel, et al., 2013) o la implicación del alumnado en el proceso de aprendizaje (Inglés et al., 2014). Sobre el papel que juega el concepto que las personas tienen de sí mismas sobre la implicación escolar, los resultados de este estudio sugieren que una buena autopercepción global predice niveles altos en la implicación conductual y emocional, pero no incide significativamente sobre la implicación cognitiva, ubicándose en la misma línea que el estudio de Veiga, García, Reeve, Wentzel y García (2015). Asimismo, estos resultados concuerdan con lo encontrado en la bibliografía previa (Rodríguez-Fernández, et al., 2012; Rodríguez-Fernández, Ramos-Díaz, Madariaga, et al., 2016; Rodríguez-Fernández, Ramos-Díaz, Fernández-Zabala, et al., 2016), pero matizan tales resultados, pues en ellos únicamente se tenía en cuenta la medida general de la implicación escolar, no sus dimensiones. Ahora bien, a pesar de este hallazgo se necesita seguir avanzando en esta relación, por lo que en un futuro convendría ir más allá de la medida global del autoconcepto incluyendo la escala de autoconcepto académico, dado que lo que se explora es la implicación escolar, o incluso ver si tales relaciones se ven modificadas al incluir otros dominios del autoconcepto como el personal o el social.

En lo que respecta a la relación entre resiliencia e implicación escolar son escasos los estudios que la analizan (Jones y Lafreniere, 2014; Ungar y Liebenberg, 2013), a pesar de que al amparo de la psicología positiva han surgido investigaciones relacionando la resiliencia con otras variables educativas como el clima motivacional del aula (Alonso-Tapia, et al., 2013) o el rendimiento académico en estudiantes adolescentes (Gaxiola, et al., 2013). Los resultados de esta investigación comprueban la influencia directa de la resiliencia en todas las dimensiones de la implicación escolar (conductual, emocional y cognitiva). Estos resultados son consistentes con los obtenidos en uno de los escasos estudios que aúnan resiliencia e implicación escolar (Jones y Lafreniere, 2014), que encuentra una relación positiva entre ambas variables. 
En síntesis, el modelo de regresión múltiple sometido a verificación empírica en esta investigación ha permitido constatar el reconocimiento de la resiliencia y el autoconcepto como atributos psicológicos decisivos en la predicción de la implicación escolar de los adolescentes de ambos sexos, a excepción de la implicación cognitiva, que no viene determinada por el autoconcepto. La relación más destacada es la que mantiene el autoconcepto con las facetas conductual y emocional de la implicación escolar, mientras que la influencia de la resiliencia es especialmente relevante en la implicación cognitiva. Se comprueba también una correspondencia entre el apoyo familiar y el ajuste escolar; en cambio, el clima social de las amistades adolescentes parece predecir positivamente la implicación emocional y negativamente la implicación conductual y la implicación cognitiva.

Por tanto, si para los escolares adolescentes los componentes más determinantes de la implicación escolar son la resiliencia y el autoconcepto, los resultados poseen implicaciones educativas que permiten sugerir la importancia de fomentar el desarrollo psicosocial humano en contextos educativos a través de programas de intervención psicológica que promocionen el afrontamiento positivo de la adversidad y la aceptación de uno mismo. También desde la escuela debe prestarse atención a la mejora de las relaciones sociales para lograr un buen ajuste personal. En este sentido, la relación diferenciada que ejercen el contexto familiar y el contexto de las amistades en la adaptación escolar adolescente suscita varios interrogantes, ya que mientras la percepción de soporte familiar se relaciona positivamente con la implicación escolar, el sentimiento de pertenencia y acogida dentro del grupo juvenil de amistades se muestra asociado con el sentimiento de pertenencia a la escuela, pero parece determinar negativamente los aspectos de participación e inversión en la tarea de aprendizaje. Esta constatación seguramente puede ser reflejo de una realidad sociológica determinada que debería ser contrastada con datos de otros contextos sociológicos.

En futuros estudios debería mantenerse el foco de atención sobre dicho aspecto, dada la decisiva influencia que las relaciones de amistad ejercen en el periodo de la adolescencia. Pero también se deberían incluir medidas más concretas del apoyo de los amigos o del apoyo de la familia, como por ejemplo el estatus sociométrico del alumno en su grupo de clase o los estilos educativos parentales, de forma que se pueda comprobar si, al incluir estas medidas más exhaustivas de ambos tipos de apoyo, se ayuda a explicar en mayor medida la implicación escolar. Ahora bien, siguiendo la idea planteada al inicio de este apartado de considerar diferentes elementos al mismo tiempo (contextuales, psicológicos y conductuales) que ayuden a dar una explicación más real de la conducta adolescente (Casas, 2011), será 
necesario probar el planteamiento teórico de que el apoyo social percibido incide sobre la implicación escolar de forma indirecta a través de factores psicológicos (resiliencia y autoconcepto). Para ello, se requerirá de análisis estadísticos más potentes como los modelos de ecuaciones estructurales, que permitan examinar detalladamente las intrincadas y complejas relaciones entre todas estas variables. 


\section{REFERENCIAS BIBLIOGRÁFICAS}

Alonso-Tapia, J., Nieto, C., \& Ruíz, M. A. (2013). Measuring subjective resilience despite adversity due to family, peers and teachers. The Spanish Journal of Psychology, 16, 1-13. doi:10.1017/ sjp.2013.33

Appleton, J. J. (2012). Systems consultation: Developing the assessmentto-intervention link with the Student Engagement Instrument. En S. L. Christenson, A. L. Reschly, C. Wylie, S. L. Christenson, A. L. Reschly \& C. Wylie (Eds.), Handbook of research on student engagement (pp. 725-741). New York, NY, US: Springer Science + Business Media. doi:10.1007/978-1-46142018-7_35

Appleton, J. J., Christenson, S. L., y Furlong, M. J. (2008). Student engagement with school: Critical conceptual and methodological issues of the construct. Psychology in the Schools, 45(5), 369-386. doi:10.1002/pits.20303

Blumenfeld, P., Modell, J., Bartko, W., Secada, W. G., Fredricks, J. A., Friedel, J., \& Paris, A. (2005). School engagement of inner-city students during middle childhood. En C. R. Cooper, C. Coll, W. Bartko, H. Davis \& C. Chatman (Eds.), Developmental pathways through middle childhood: Rethinking contexts and diversity as resources (pp. 145-170). Mahwah, NJ US: Lawrence Erlbaum Associates Publishers.

Bobes, J., Bascaran, M. T., García-Portilla, M. P., Bousoño, M., Sáiz, P. A. y Wallance, D. H. (2001). Banco de instrumentos básicos de psiquiatría clínica. Barcelona: Psquiatría ED.

Bronfenbrenner, U. (1986). Ecology of the family as a context for human development: Research perspectives.
Developmental Psychology, 22(6), 723742. doi:10.1037/0012- 1649.22.6.723

Campbell-Sills, L., Cohan, S. L., y Stein, M. B. (2006). Relationship of resilience to personality, coping, and psychiatric symptoms in young adults. Behaviour Research and Therapy, 44(4), 585-599. doi:10.1016/j.brat.2005.05.001

Casas, F. (2011). Subjective social indicators and child and adolescent wellbeing. Child Indicators Research, 4(4), 555-575. doi:10.1007/s12187-010-9093-z

Christenson, S. L., Reschly, A. L., \& Wylie, C. (2012). Handbook of research on student engagement. New York, NY, US: Springer Science. doi:10.1007/9781-4614-2018-7

Cohen, S. (2004). Social relationships and health. American Psychologist, 59(8), 676684. doi:10.1037/0003-066X.59.8.676

Connor, K. M., \& Davidson, J. T. (2003). Development of a new resilience scale: The Connor-Davidson Resilience Scale (CD-RISC). Depression and Anxiety, 18(2), 76-82. doi:10.1002/da.10113.

Fergus, S., \& Zimmerman, M. A. (2005). Adolescent resilience: A framework for understanding healthy development in the face of risk. Annual Review of Public Health, 26, 399-419. doi:10.1146/ annurev.pubhealth.26.021304.144357

Fernández-Zabala, A., Goñi, E., Rodríguez-Fernandez, A., y Goñi A. (2015). Un nuevo cuestionario en castellano con escalas de las dimensiones del autoconcepto. Revista Mexicana de Psicología, 32(2), 149-159.

Fletcher, D., \& Sarkar, M. (2013). Psychological resilience: A review and critique of definitions, concepts, and theory. European Psychologist, 18(1), 12-23. doi:10.1027/1016-9040/a000124 
ARANTZAZU RODRÍGUEZ-FERNÁNDEZ, ESTIBALIZ RAMOS-DÍAZ, IKER ROS MARTÍNEZ DE LAHIDALGA

Fredricks, J. A., Blumenfeld, P. C., Friedel, J., \& Paris, A. (2005). School engagement. En K. A. Moore y L. Lippman (Eds.), Conceptualizing and measuring indicators of positive development: What do children need to flourish (pp. 305-321). New York: Kluwer Academic/Plenum Press.

Fredricks, J. A., Blumenfeld, P. C., \& Paris, A. H. (2004). School engagement: Potential of the concept, state of the evidence. Review of Educational Research, 74(1), 59-109. doi:10.3102/00346543074001059.

Fredricks, J. A., \& McColskey, W. (2012). The measurement of student engagement: A comparative analysis of various methods and student self-report instruments. In S. L. Christenson, A. L. Reschly, C. Wylie, S. L. Christenson, A. L. Reschly, \& C. Wylie (Eds.), Handbook of research on student engagement (pp. 763-782). New York, NY, US: Springer. doi:10.1007/978-1-46142018-7_37

Fried, L., \& Chapman, E. (2012). An investigation into the capacity of student motivation and emotion regulation strategies to predict engagement and resilience in the middle school classroom. The Australian Educational Researcher, 39(3), 295-311. doi: 10.1007/ s13384- 011-0049-1.

Gaixola, J. G., Lugo, S. G., y Villa, E. G. (2013). Autorregulación, resiliencia y metas educativas: Variables protectoras del rendimiento académico de bachilleres. Revista Colombiana de Psicología, 22(2), 241-252.

Gaixola, J. G., Lugo, S. G., Guedea, M. D., y Villa, E. G. (2013). Autorregulación, metas y rendimiento académico en bachilleres con disposiciones resilientes y no resilientes. Revista Interamericana de Psicología, 47(1), 71-82.
Garcia-Reid, P., Reid, R. J., \& Peterson, N. A. (2005). School engagement among Latino youth in an urban middle school context valuing the role of social support. Education and Urban Society, 37(3), 257-275. doi: $10.1177 / 0013124505275534$

Glanville, J. L., \& Wildhagen, T. (2007). The measurement of school engagement: Assessing dimensionality and measurement invariance across race and ethnicity. Educational and Psychological Measurement, 67(6), 1019-1041. doi:10.1177/0013164406299126

González, A., y Verónica-Paoloni, P. (2014). Implicación y rendimiento en Física: el papel de las estrategias docentes en el aula, y el interés personal y situacio. Revista de Psicodidáctica, 20(1). doi: http:10.1387/RevPsicodidact.11370

Gutiérrez, M., y Romero, I. (2014). Resiliencia, bienestar subjetivo y actitudes de los adolescentes hacia el consumo de drogas. Anales de Psicología, 30(2), 608-619. doi: http://dx.doi.org/10.6018/ analesps.30.2.148131

Huang, C. (2011). Self-concept and academic achievement: A meta-analysis of longitudinal relations. Journal of School Psychology, 49(5), 505-528. doi:10.1016/j.jsp.2011.07.001

Inglés, C., Martínez-Monteagudo, M., García-Fernández, J., Valle, A., \& Castejón, J. (2014). Goal orientation profiles and self-concept of secondary school students. Journal of Psychodidactics, 20(1). doi:10.1387/RevPsicodidact.1023

Jones, G., \& Lafreniere, K. (2014). Exploring the role of school engagement in predicting resilience among bahamian youth. Journal of Black Psychology, 40(1), 47-68. doi: 10.1177/0095798412469230 
Jorgensen, I. E., \& Seedat, S. (2008). Factor structure of the Connor-Davidson resilience scale in South African adolescents. International Journal of Adolescent Medicine and Health, 20(1), 23-32. doi: 10.1515/ijamh.2008.20.1.2

Juvonen, J., Espinoza, G., \& Knifsend, C. (2012). The role of peer relationships in student academic and extracurricular engagement. En S. L. Christenson, A. L. Reschly \& C. Wylie (Eds.), Handbook of research on student engagement (pp. 387-401). New York, NY US: Springer Science + Business Media. doi:10.1007/978-1-4614-2018-7_18

Kristjánsson, K. (2012). Positive psychology and positive education: Old wine in new bottles?. Educational Psychologist, 47(2), 86-105. doi:10.10080/00461 2520.2011.610678

Lam, S., Jimerson, S., Kikas, E., Cefai, C., Veiga, F. H., Nelson, B.,... Zollneritsch, J. (2012). Do girls and boys perceive themselves as equally engaged in school? The results of an international study from 12 countries. Journal of School Psychology, 50(1), 77-94. doi:10.1016/j.jsp.2011.07.004

Lam, S., Wong, B. H., Yang, H., \& Liu, Y. (2012). Understanding student engagement with a contextual model. En S. L. Christenson, A. L. Reschly \& C. Wylie (Eds.), Handbook of research on student engagement (pp. 403-419). New York, NY US: Springer Science. doi:10.1007/978-1-4614-2018-7_19

Lawson, M., \& Lawson, A. (2013). New Conceptual Frameworks for Student Engagement Research, Policy, and Practice. Review of Educational Research, 83(3), 432-479. doi: $10.3102 / 0034654313480891$

Li, Y., \& Lerner, R. M. (2011). Trajectories of school engagement during adolescence: Implications for grades, depression, delinquency, and subs- tance use. Developmental Psychology, 47(1), 233-247. doi:10.1037/ a0021307

Luthar, S. S., Cicchetti, D., \& Becker, B. (2000). The construct of resilience: A critical evaluation and guidelines for future work. Child Development, 71(3), 543-562. doi:10.1111/1467- 8624.00164

Masten, A. S. (2014). Invited commentary: Resilience and positive youth development frameworks in developmental science. Journal of Youth and Adolescence, 43(6), 1018-1024. doi:10.1007/s10964-014-0118-7

Moreno, J. A., y Vera, J. A. (2011). Modelo causal de la satisfacción con la vida en estudiantes adolescentes. Revista de Psicodidáctica, 16(2), 367-380.

Motti-Stefanidi, F., \& Masten, A. S. (2013). School success and school engagement of immigrant children and adolescents: A risk and resilience developmental perspective. European Psychologist, 18(2), 126-135. doi:10.1027/1016-9040/a000139

Perdue, N. H., Manzeske, D. P., \& Estell, D. B. (2009). Predicting school commitment at grade five: Exploring the role of students' relationships with peers and teachers from grade three. Psychology in the Schools, 46, 10841097. doi: 10.1002/pits.20446

Preckel, F., Niepel, C., Schneider, M., \& Brunner, M. (2013). Self-concept in adolescence: A longitudinal study on reciprocal effects of self-perceptions in academic and social domains. Journal of Adolescence, 36(6), 1165-1175. doi:10.1016/j.adolescence.2013.09.001

Ramos-Díaz, E., Rodríguez-Fernández, A., Fernández-Zabala, A., Revuelta, L., y Zuazagoitia, A. (2016). Apoyo social percibido, autoconcepto e implicación escolar de estudiantes adolescentes. Revista de Psicodidáctica, 21(2). doi: 10.1387/RevPsicodidact.14848 
ARANTZAZU RODRÍGUEZ-FERNÁNDEZ, ESTIBALIZ RAMOS-DÍAZ, IKER ROS MARTÍNEZ DE LAHIDALGA

Rodríguez-Fernández, A., Droguett, L., y Revuelta, L. (2012). Ajuste escolar y personal en la adolescencia: El papel del autoconcepto académico y del apoyo social percibido. Revista de Psicodidáctica, 17(2). doi: 0.1387/Rev. Psicodidact.3002

Rodríguez-Fernández, A., Ramos-Díaz, E., Fernández-Zabala, A., Goñi, E., Esnaola, I., \& Goñi, A. (2016). Contextual and psychological variables in a descriptive model of subjective well-being and school engagement. International Journal of Clinical and Health Psychology. doi: 10.1016/j. ijchp.2016.01003

Rodríguez-Fernández, A., Ramos-Díaz, E., Madariaga, J. M., Arribillaga, A., \& Galende, N. (2016). Steps in the construction and verification of an explanatory model of psychosocial adjustment. European Journal of Education and Psychology, 9(1), 20-28. doi:10.1016/j.ejeps.2015.11.002

Rodríguez-Fernández, A., Ramos-Díaz, E., Ros, I., Fernández-Zabala, A., y Revuelta, L. (2015). Resiliencia e implicación escolar en función del sexo y del nivel educativo en educación secundaria. Aula Abierta. doi:/10.1016/j. aula.2015.09.001

Rutter, M. (2007). Resilience, competence, and coping. Child Abuse and Neglect, 31(3), 205-209. doi:10.1016/j. chiabu.2007.02.001

Shavelson, R. J., Hubner, J. J., \& Stanton, G. C. (1976). Self-concept: Validation of construct interpretations. Review of Educational Research, 46(3), 407-441. doi:10.2307/1170010

Simons-Morton, B., \& Chen, R. (2009). Peer and parent influences on school engagement among early adolescents. Youth and Society, 41(1), 3-25. doi:10.1177/0044118X09334861
Toland, J., \& Carrigan, D. (2011). Educational psychology and resilience: New concept, new opportunities. School Psychology International, 32(1), 95106. doi:10.1177/0143034310397284

Ungar, M. (2011). The social ecology of resilience: Addressing contextual and cultural ambiguity of a nascent construct. American Journal of Orthopsychiatry, 81(1), 1-17. doi:10.1111/ j.1939-0025.2010.01067.x

Ungar, M., y Liebenberg, L. (2013). Ethnocultural factors, resilience, and school engagement. School Psychology International, 34(5), 514-526. doi:10.1177/0143034312472761

Veiga, F. H., Burden, R., Appleton, J., do Céu Taveira, M., y Galvao, D. (2014). Envolvimiento de los estudiantes en la escuela: conceptualización y relaciones con variables personales y rendimiento académico. Una revisión de la literatura. Revista de Psicología y Educación, 9(1), 29-47.

Veiga, F. H., Carvalho, C., Almeida, A., Taveira, C., Janeiro, I., Baía, S.,... Caldeira, S. (2012). Students' engagement in schools: differentiation and promotion. En M. F. Patrício, L. Sebastião, J. M. Justo \& J. Bonito (Eds.), Da exclusão à excelência: Caminhos organizacionais para a qualidade da educação (pp. 117123). Montargil: Associação da Educação Pluridimensional e da Escola Cultural.

Veiga, F., García, F., Reeve, J., Wentzel, K., \& García, Ó. (2015). When adolescents with high self-concept lose their engagement in school. Revista de Psicodidáctica, 20(2), 305-320. doi: 10.1387/RevPsicodidact.12671

Yu, X. N., Lau, J. T., Mak, W. W., Zhang, J., \& Lui, W. W. (2011). Factor structure and psychometric properties of the Connor-Davidson Resilience Scale among Chinese adolescents. Comprehensive Psychiatry, 52(2), 218-224. doi: 10.1016/j.comppsych.2010.05.010 


\section{PERFIL ACADÉMICO Y PROFESIONAL DE LOS AUTORES}

Arantzazu Rodríguez Fernández. Doctora en psicología. Profesora del Departamento de Psicología Evolutiva y de la Educación de la Universidad del País Vasco (UPV/EHU).

Estibaliz Ramos-Díaz. Doctora en psicología. Profesora del Departamento de Psicología Evolutiva y de la Educación de la Universidad del País Vasco/Euskal Herriko Unibertsitatea (UPV/EHU).

Iker Ros. Doctor en psicopedagogía, licenciado en geografía e historia, licenciado en educación física, y diplomado en magisterio. Profesor del Departamento de Didáctica de la Expresión Musical, Plástica y Corporal de la Universidad del País Vasco/Euskal Herriko Unibertsitatea (UPV/EHU).

Ana Zuazagoitia-Rey Baltar. Doctora en Ciencias de la Actividad Física y del Deporte. Profesora del Departamento de Didáctica de la Expresión Musical, Plástica y Corporal de la Universidad del País Vasco/ Euskal Herriko Unibertsitatea (UPV/EHU).

Dirección de los autores: Facultad de Educación y Deporte Juan Ibáñez de Santo Domingo 1 01006 Vitoria-Gasteiz (Álava)

E-mail: arantzazu.rodriguez@ehu.eus estibaliz.ramos@ehu.eus iker.ros@ehu.eus ana.zuazagoitia@ehu.eus

Los autores de este estudio son miembros del grupo Consolidado de Investigación IT934-16 del Sistema Universitario Vasco. El estudio se enmarca como parte de los proyectos de investigación PPG17/61 y EHUA 15/15 de la Universidad del País Vasco/Euskal Herriko Unibertsitatea.

Fecha recepción del artículo: 16. Diciembre. 2015

Fecha modificación del artículo: 13. Febrero. 2016

Fecha aceptación del artículo: 16. Febrero. 2016

Fecha revisión para publicación: 31. Mayo. 2017 